\title{
Variations of the granulation related to the solar cycle and with respect to its position on the solar disk
}

\author{
R. Muller ${ }^{1}$, A. Hanslmeier ${ }^{2}$, and M. Saldaña-Muñoz ${ }^{2}$ \\ 1 Laboratoire d'Astrophysique de l'Observatoire Midi-Pyrénées, Université Paul Sabatier, CNRS, Observatoire du Pic du Midi, \\ 57 avenue d'Azereix, BP 826, 65008 Tarbes Cedex, France \\ e-mail: muller@ast.obs-mip.fr \\ 2 Institut für Physik, Karl-Franzen Universität Graz, Universitätsplatz 5, 8010 Graz, Austria \\ e-mail: arnold.hanslmeier@uni-graz.at
}

Received 31 July 2007 / Accepted 7 September 2007

\begin{abstract}
Aims. We investigate variations in the scale and contrast of the solar granulation related to the solar cycle during the period 1978-1993. Furthermore, as a by-product, we have detected a variation with respect to the solar longitude, along the solar equator.

Methods. All images were taken on film with the $50 \mathrm{~cm}$ refractor of the Pic du Midi Observatory, under excellent seeing conditions. Scale and contrast were derived from power spectra computed with digitized images. This simple and robust statistical method allowed us to get reliable results, independent of any image-processing parameter.

Results. The contrast of the solar granulation varies nearly in phase with the solar cycle, being smaller at the periods of solar maximum. But we detected no corresponding variation in the scale; if there is one, it must be of low amplitude and masked by a spatial variation in the scale with respect to the position on the solar equator, which amounts to $3 \%$ rms. The contrast also varies with the position on the equator, in phase with the scale: where the scale is larger, the contrast is higher too. The amplitude of this spatial variation in the granulation scale is $9 \%$; large photometric uncertainties did not allow us to quantify the amplitude of the contrast variation.
\end{abstract}

Key words. Sun: activity - Sun: granulation - Sun: magnetic fields

\section{Introduction}

More than two decades ago, Macris \& Rösch (1983), and a little later Macris et al. (1984), reported that the mean distance between granule centres decreases with increasing activity, with an amplitude as large as $10 \%$. But the set of images they used was not homogeneous, because they were taken with two different refractors, 38 and $50 \mathrm{~cm}$ of diameter, at different wavelengths and under varying seeing conditions at the Pic du Midi Observatory, between 1966 and 1976. In addition, one image, taken with the $60 \mathrm{~cm}$ Solar Tower Telescope at Sac Peak Observatory, was included. The decrease in granule size with increasing activity was confirmed later by Roudier (1986), who counted the number of granules per surface unit on images of higher homogeneity taken between 1973 and 1985. However, the variation does not appear so clearly in the counting made by Muller (1988), with a new set of homogeneous images taken between 1977 and 1988, also from the Pic du Midi Observatory. In all of these investigations, granules were identified visually. This induces some uncertainties, because it is difficult to keep the same granule identification criterion for all images.

Here, we present new results derived from analysis of a much more homogeneous set of images, all taken with the $50 \mathrm{~cm}$ refractor at the Pic du Midi Observatory between 1978 and 1993, under excellent seeing conditions. The images were analysed with simple statistical methods, that allowed us to control the various sources of uncertainties.
It is important to know whether the properties of the solar granulation change during the solar activity cycle, because they may reflect changes in the physical properties in subphotospheric layers and changes in convective efficiency, probably under the effect of a variable magnetic field. Furthermore, granulation changes would provide an additional constraint to solar models that attempt to reproduce observed solar cycle-related variations in the global parameters, like the total solar irradiance, the solar radius or the photospheric temperature. They also attempt to reproduce the well known variations in the frequencies of $\mathrm{f}$ and $\mathrm{p}$-mode oscillations; for example, variations in turbulence and magnetic field are often invoked to explain p-mode frequency shifts during the solar cycle (Basu \& Antia 2000; Khun 2000; Houdek et al. 2001; Li et al. 2003; Dziembowski \& Goode 2005). Variations in turbulence (together with variations of magnetic field) are also included in models that attempt to derive changes in the solar diameter from observed shifts in f-modes related to the solar cycle (Li et al. 2003; Sofia et al. 2005).

\section{Observations and image processing}

The same, very simple optical set up was used over the years: it consisted of a reimaging lens and an interference filter $(6 \mathrm{~nm}$ wide, centred at $575 \mathrm{~nm}$ ). However, the data set was not perfectly homogeneous, because the image scale was not kept constant, varying between 0 ' 08 and 0 !' 10 per pixel, and because the film calibrations, which are used to transform film densities into 

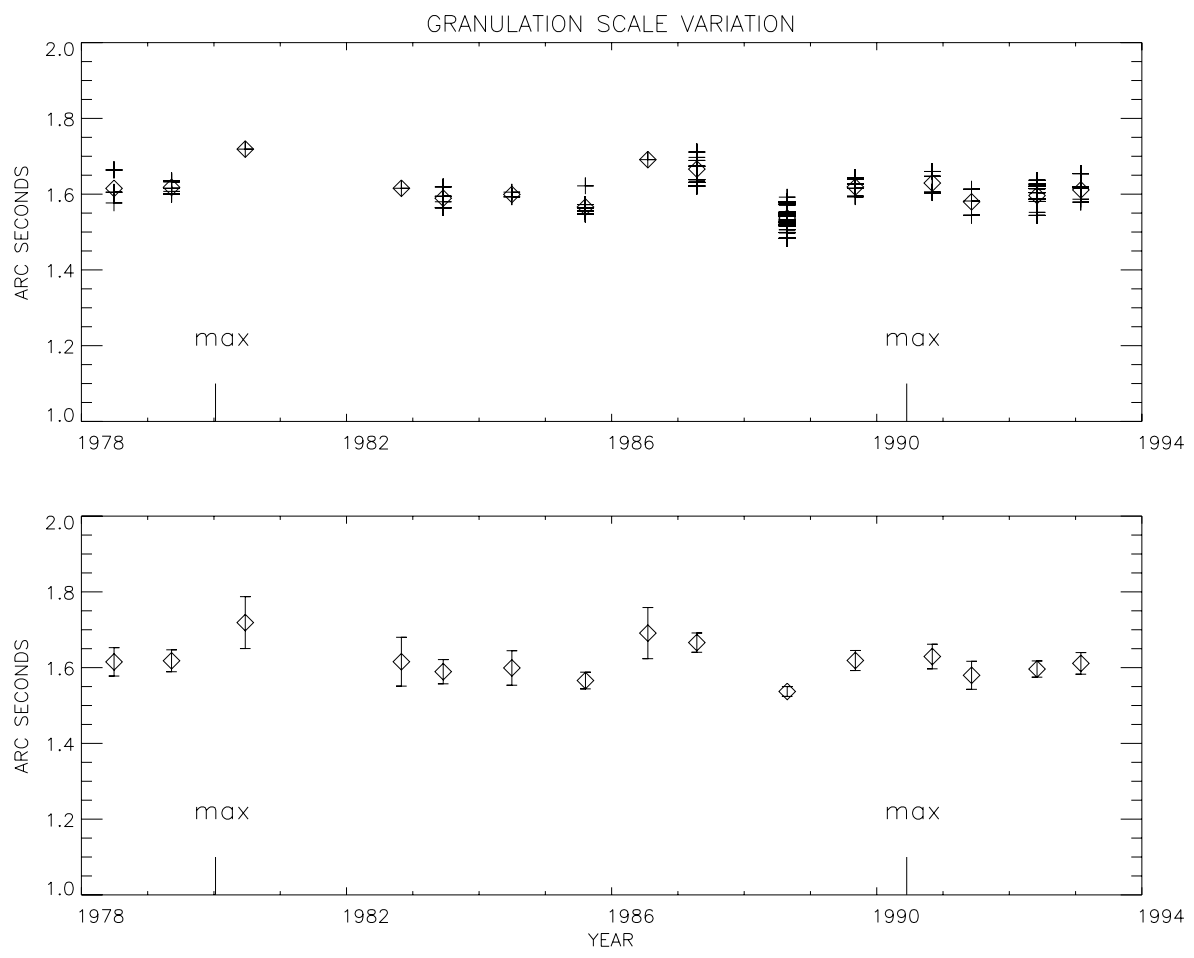

Fig. 1. Variation in the granulation scale, defined as the median wavenumber of the power spectrum converted into arcseconds, between 1978 and 1993; upper panel: each cross represents one image, field of view: $65^{\prime \prime} \times 65^{\prime \prime}$; lower panel: mean values and statistical error bars. The positions of the solar maxima are indicated.

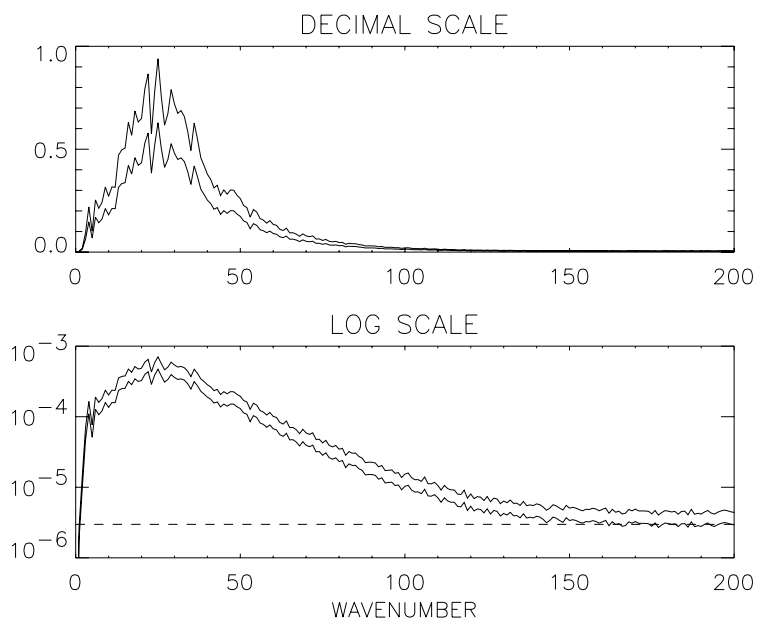

Fig. 2. Power spectra of one granulation image, computed with two different film calibrations. They are plotted in decimal scale in the upper panel and in logarithmic scale in the lower panel.

solar intensities, were inaccurate. In fact, film calibrations are the main source of uncertainties for granulation contrast measurements. Some possible slight residual seeing differences among images proved to be negligible.

All images were taken at the disk centre or at close proximity, to avoid foreshortening effects. They belong either to time series or to sets of images taken at different positions. The absence of abnormal granulation in the white-light images insures that they were taken in quiet-sun areas, away from remnants of active regions and from the active network. Inspection of the associated G-band images, often available, confirmed that the observed areas were very quiet. The images were digitized with the machine MAMA at the Observatoire de Paris.

The philosophy adopted for processing our particular set of images, was to use techniques that have a minimum effect on the results. That is why the granulation scale and contrast

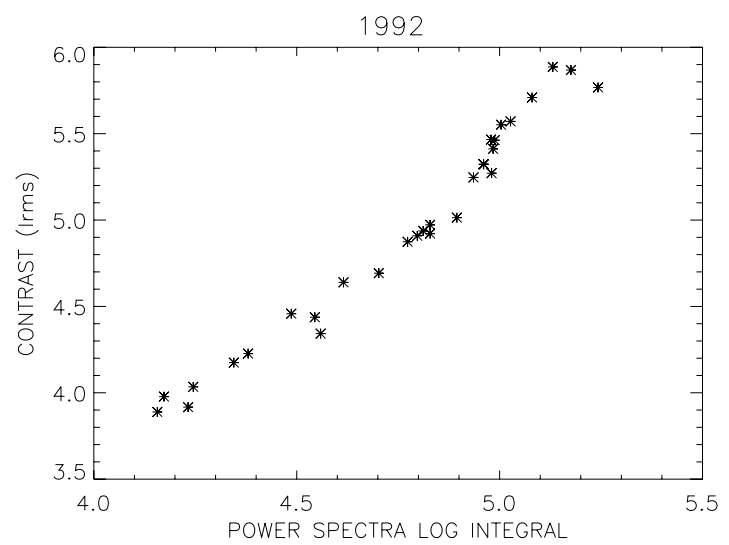

Fig. 3. Relation between the square root of the integral of the power spectra (contrast) and of their integral computed in logarithmic scale (log integral) for the images taken in 1992.

variations were simply derived from power spectra, which do not require any image pre-processing that could affect the results. More information would be provided by granule histograms derived from granule segmentation (segmentation determines the contour of granules). But it depends on several parameters (filter parameters, cut-off level, pixel scale), that are difficult to control and to keep constant for our set of images of different scales, contrasts, and film noise.

\section{Results}

\subsection{Granulation scale variation}

The median wavenumber,

$K_{\mathrm{m}}=\int_{0^{\prime \prime} 5}^{3^{\prime \prime} 5} k P(k) \mathrm{d} k / \int_{0^{\prime \prime} 5}^{3^{\prime \prime} 5} P(k) \mathrm{d} k$,

where $k$ is the wavenumber and $P(k)$ the $2 \mathrm{D}$ power spectrum, was used to measure the granulation scale. The integration was 

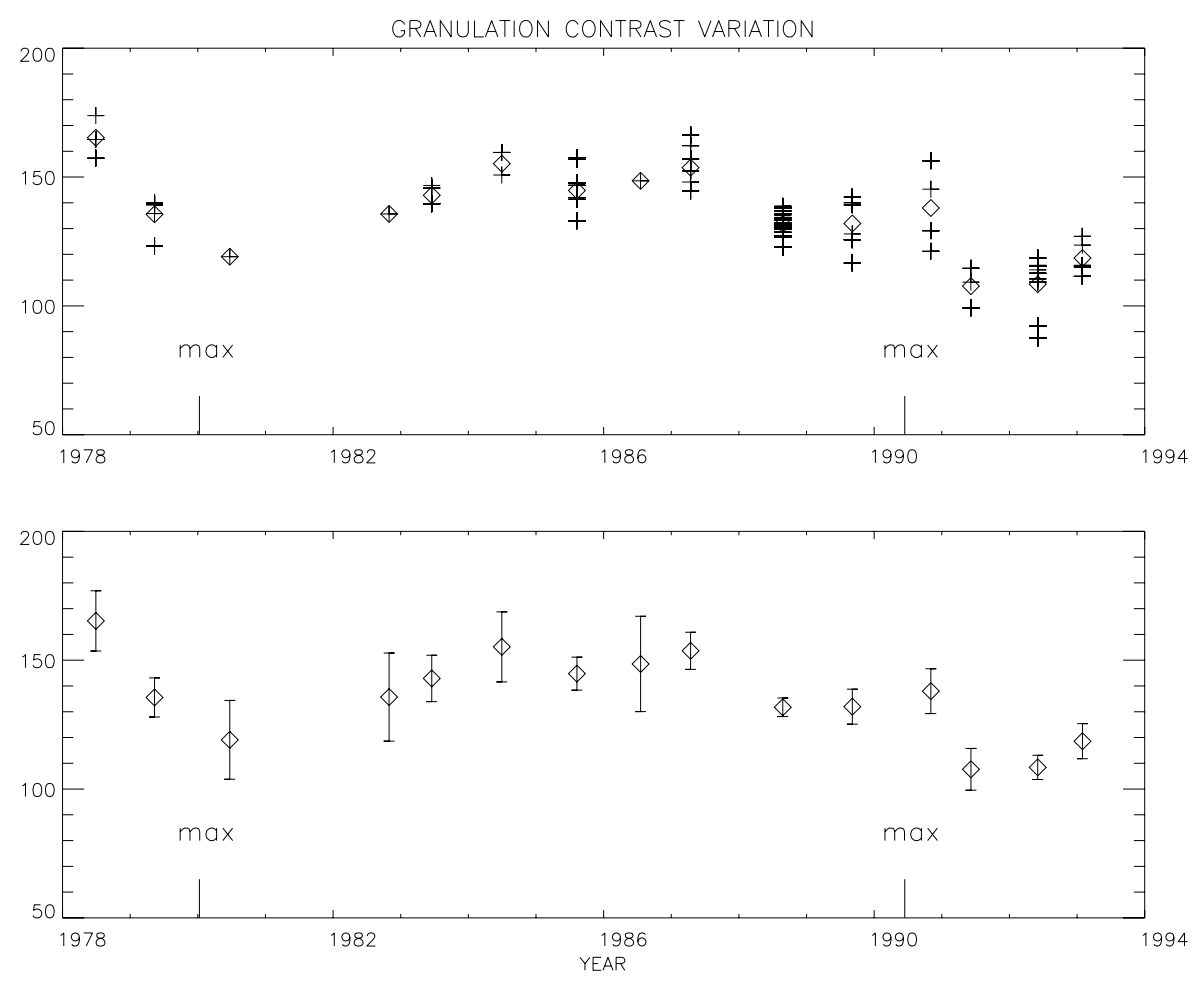

Fig. 4. Variation in the granulation contrast, defined as the integral of the logarithm of the power spectrum, above the noise level, in the granulation range (0.'5-3'.5, between 1978 and 1993. Upper panel: each cross represents one image, field of view: $65^{\prime \prime} \times 65^{\prime \prime}$; lower panel: mean values and statistical error bars.

restricted to the range of granule sizes: [0.5-3'.5] in order to remove high frequency noise and large scale fluctuations not of a granular origin (associated to mesogranules, supergranules, 5-min oscillations, and illumination inhomogeneities). Its variation between 1978 and 1993 is plotted in Fig. 1. Each cross in the upper panel is the median wavenumber of one granulation image (field of view $700 \times 700$ pixels, or about $65^{\prime \prime} \times 65^{\prime \prime}$, depending on the image scale). The images taken on the same day or on a few consecutive days belong to what we call an "observation". The diamonds represent the average $K_{\mathrm{m}}$ of each observation. Several interesting features are noticeable in Fig. 1: 1) There is no detectable variation in the granulation scale related to the solar cycle. 2) In several observations, the dispersion is large, up to $8 \%$ due, to nearly an equal part, to the evolution of the granular pattern and to spatial differences when the images were taken at various positions near the disk centre. 3) There are also large differences of $K_{\mathrm{m}}$ between observations; for example: $9 \%$ between 1987 and 1988, 7\% between 1988 and 1990 and between 1985 and 1987. These differences are much larger than the statistical error bars (Fig. 1, lower panel), which are simply derived from the maximum dispersion $(8 \%)$ and the number of images in each observation. As we have not found any instrumental reason explaining them, we come to the conclusion that they are of solar origin. Since the observations were taken at the disk centre, but at random relative to the solar longitude, they should reflect $a$ spatial variation of the granulation scale around the equator. We will come back to this point in the next section. Not surprisingly, a closely identical variation was obtained for the width of the granulation autocorrelation function.

\subsection{Granulation contrast variation}

Power spectra were used to compute the root mean square of the granulation intensity fluctuations $\delta I_{\text {rms }}$, which is the square root of the power spectra integral. For simplicity, we call it "contrast". The problem is that the amplitude of the power spectra is related to the film calibrations, which are inaccurate.
Consequently, our observations do not allow us to derive the contrasts with enough accuracy to expect to find a variation with the solar cycle. Fortunately, the integration of the power spectra in the logarithmic scale above noise level is independent of film calibration: when computed with different calibrations, the power spectra plotted in the logarithmic scale are just shifted, compared to each other, in the $y$-direction of the plot, without any deformation (Fig. 2). Thus, the integral computed in the logarithmic scale above the noise level (integral of $\log$ (Power) $\log$ (Noise), which we call "log integral" for simplicity) is independent of the calibration. The integration is made in the granulation range between [0, $\left.5-33^{\prime \prime} 5\right]$. For each observation, there is a close linear relationship between the contrast and the log integral (Fig. 3). Consequently, the log integral can be used as a proxy to investigate contrast variations. For that reason, "log integral" is simply called "contrast" in the following. It can be noticed that the logarithmic integration of the power spectra artificially increases the contribution of the smaller scales to the granulation contrast, as shown by Fig. 2 .

The variation in the log integral (contrast proxy) for the period 1978 to 1993 is plotted in Fig. 4. A variation nearly in phase with the solar cycle clearly appears with less contrast in the periods of sunspot maxima, and it seems that there is a delay of a year. Just as with $K_{\mathrm{m}}$ (Fig. 1), the dispersion of the granulation contrast is large in several observations, up to $20 \%$. The reasons are the same as for $K_{\mathrm{m}}$ : granule evolution and different positions of observation near the disk centre. The difference in contrast between the maxima of activity in 1980 and 1991 and the minima of 1986 is $15 \%$ and $25 \%$ respectively, significantly larger than the statistical error bars (Fig. 4, lower panel). As we do see no instrumental reason for such large differences, it means that the observed contrast variation is certainly real.

Moreover, when one compares the variations in contrast and scale $\left(K_{\mathrm{m}}\right)$, the trends over short periods of time are very similar (Fig. 5). They both increase between 1991 and 1993 and between 1988 and 1990, and there are jumps between 1990 and 1991 and between 1987 and 1988 . The trends are not very different in the 

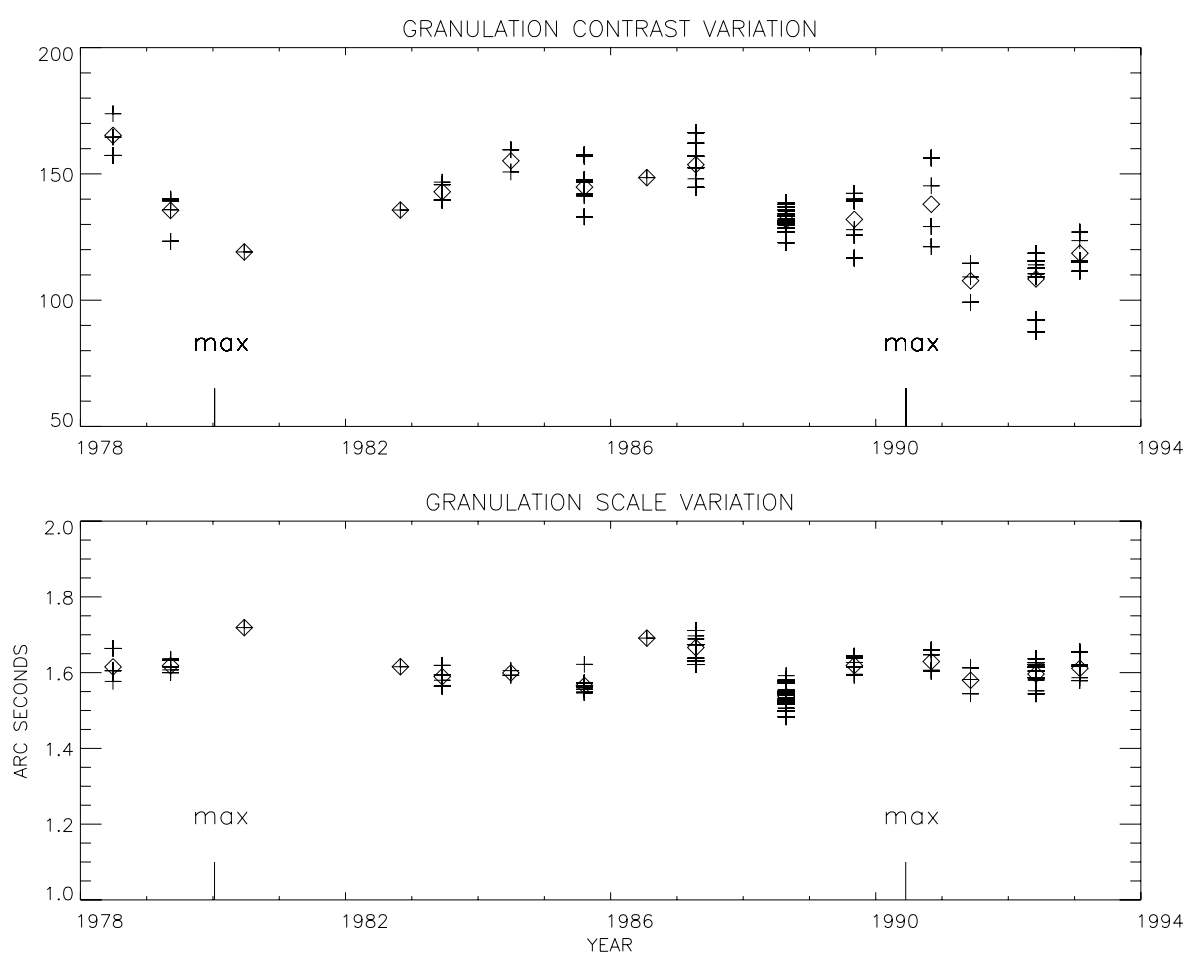

Fig. 5. Comparison of the contrast (upper panel) and of the scale (lower panel) variations of the solar granulation.

period 1982-1987, if we take into account the rise in contrast related to the decline in activity in the same period. However, the trends in the slopes are opposite between 1978 and 1980, but this is the less accurate part of the variation, since the number of available images is small. Moreover, the image in 1980 was taken with a different optical configuration, as a beam splitter was placed in the beam and the reimaging lens had a different focal length from the one used in the normal set up. To derive the image scale, we simply used the nominal focal length value provided by the vendor, which might be a little different from the real one, which was not measured. The pixel size in arcseconds for 1980 may thus be slightly incorrect, compared to the scales of the other observations. It must be noted that the contrast (and its time variation) is nearly independent of the pixel scale, while the median wavenumber, expressed in arcseconds like in Fig. 1, is nearly inversely proportional to it, since it is the wavenumber derived from Eq. (1), simply divided by the scale.

The similarity of the trends over short periods of time of the contrast and scale variations, means that, when one compares the granulation at two different positions on the equator, where the contrast is higher, the scale is larger. Thus, firstly, along the equator, the scale and the contrast of the solar granulation vary in phase; secondly, the long-term variation in the contrast, observed at the disk centre, appears to be a combination of two components: a time variation in antiphase with the solar cycle superimposed on a spatial variation around the solar equator, with much weaker amplitude. When one divides the images into four subfields, the difference in scale and contrast in each subfield is very small. As the size of the subfields $\left(32^{\prime \prime} \times 32^{\prime \prime}\right.$ in average) is smaller than that of a typical supergranule, we can conclude that the period of the spatial variation is larger than that of a supergranule. One may have an idea of the period by looking at Fig. 5 in more detail. In 1983 and in 1993, the images were taken over three and two consecutive days, respectively. The Sun, while observed at the disk centre, had rotated by 26 and 13 degrees, respectively. The dispersion of the data

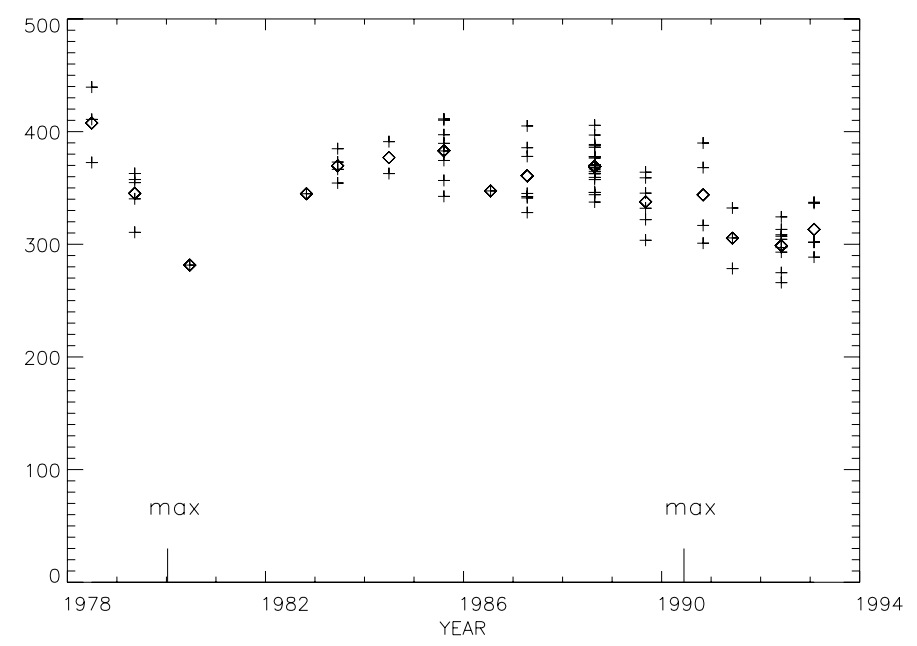

Fig. 6. Variation in the contrast of the solar granulation over the solar cycle, corrected for equatorial spatial inhomogeneities.

points is small in both cases, indicating that the period should be larger than 25 degrees. In 1992, the images were taken on July 3 and July 11: the two lower data points belong to July 3 and all the others to July 11 . The difference in the mean granulation contrast is $23 \%$, much larger than the error bars. The separation for the scale is less conspicuous, but is also larger than the error bars. From July 3 to July 11 , the Sun had rotated by 100 degrees. Therefore, we might think that the scale of the longitudinal spatial variation in the solar granulation is of the order of 100 degrees.

One can disentangle the temporal from the spatial variation in the contrast plot of Fig. 5, upper panel, by dividing it by the scale plot in the lower panel of Fig. 5. We are allowed to do that because the contrast and the scale vary nearly in phase and because the latter shows no detectable temporal variation. The result is shown in Fig. 6, where a variation in antiphase with the 

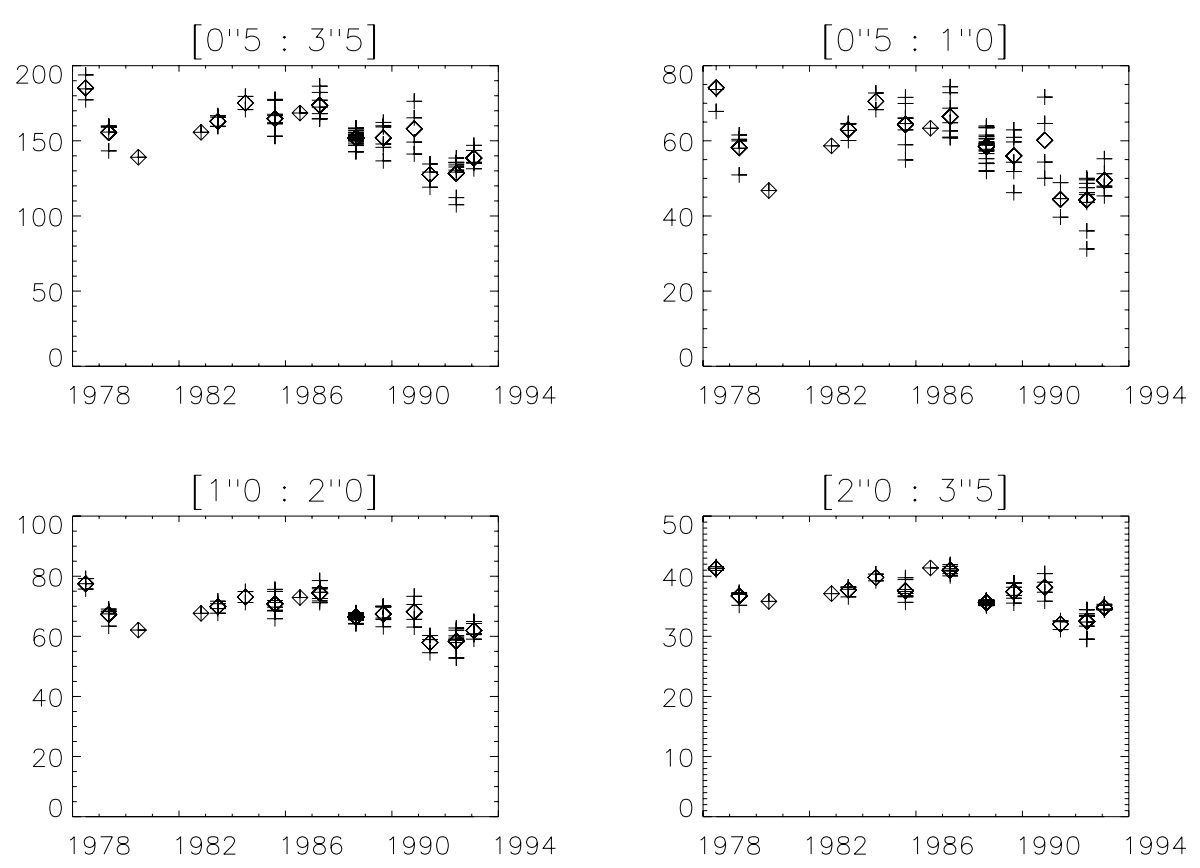

Fig. 7. Variation in the contrast of the solar granulation for various power spectra integration ranges, corresponding to small [0.'5-1.'0], medium [1.'0-2".0] and large granular features $\left[2 . .0-3{ }^{\prime \prime} 5\right]$. The upper left panel shows the variation for the full granulation range [0.'5-3'.5].

solar cycle strikingly appears. This is the variation in the contrast of the solar granulation nearly uncontaminated by the equatorial spatial variation. The slope between 1978 and 1980 is very large, but remember that this is the less accurate part of the plot and that there may be an error in the scale of the observation of 1980 .

In Fig. 7, we show the variation in the contrast with the activity cycle for different integration ranges of the power spectra: [0.'5-1'.0] corresponding to small granulation scales, [1.'0-2'.0] corresponding to intermediate scales, and [2.'0-3'.5] corresponding to large scales. It clearly appears that the contrast variation is larger for the smaller granulation scales and smaller for larger scales (the word "granulation" used here includes granular and intergranular features). Remember that the contribution of the smaller scales to the contrast variation of the granulation, shown in the upper left panel, is artificially increased, because the power spectra integration was made on a logarithmic scale.

\section{Conclusions}

Analysing a set of granulation images obtained at the Pic du Midi Observatory between 1978 and 1993, we have found that: 1) the properties of the solar granulation are changing with respect to the position on the equator (one may thus expect that the granulation is not uniform on the surface of the Sun), 2) they vary nearly in phase with the solar cycle. Concerning the spatial variation, both the granulation scale and contrast vary: where the contrast is higher, the scale is larger too. The amplitude of the scale variation is as large as $9 \%$; large photometric uncertainties do not allow us to quantify the amplitude of the associated contrast variation. The period of this variation along the equator is of the order of 100 degrees longitude. Related to the solar cycle, we have only detected a variation in the contrast, which is smaller at periods of solar maximum. We have not detected a corresponding variation in the scale; if there is one, it must be of low amplitude and masked by the spatial variation reported above, which is $3 \% \mathrm{rms}$. The amplitude of the solar cycle-related contrast variation is larger in the range of small granular features than in the range of the large ones. Unfortunately, the data does not allow us to understand why the granulation contrast is changing. Its decrease could be explained by a narrowing of the intergranular lanes. To investigate such a possibility, granule segmentation is required, but reliable results cannot be derived from our set of images because of their insufficient photometric precision and of their varying photographic film noise and pixel scale, as explained in the last paragraph of Sect. 2. Another possibility for explaining a decrease in contrast could be a filling of the intergranular lanes by an increasing number of weak brightness or unresolved magnetic elements. Analysis of the G-band images, which were taken nearly at the same time as the white-light images, will help us to answer this point; an investigation is under way.

Our results do not seem to agree with the large decrease in granulation scale with increasing activity, reported by Macris \& Rösch (1983) and by Roudier (1986), since we do not detect a scale variation of such large amplitude. It must be pointed out that Muller (1988) also did not find any clear variation in the granulation scale. In the present work, we have used a much larger sample of images: 82 , as compared to 10 used by Macris \& Rösch (1983) and 20 by Roudier (1986). Although the latter are of a little larger size, the statistical granulation noise in our investigation is about 2 times weaker. In their investigation, granules were identified visually, which is a high source of uncertainties. Moreover the same weight was given to both a small and to a large granule; as the number of small granules is much larger than the number of large ones, a much larger weight was given to small granules than in our results. Macris et al. and Roudier have only taken granules into account, while in our case, all features, including granules and intergranules, contribute to the integration of the granulation power spectra, from which contrasts and median wavenumbers were derived. In the integrations, the contribution of small granules is much smaller than that of the large ones.

The variation in the granulation contrast during the solar cycle implies that the physical properties and the turbulence beneath the surface change with varying solar activity, possibly as a result of the interaction between the convective envelope and the 
large-scale magnetic field. As a consequence, the contrast of the granulation at the surface may be reduced. But a real understanding of the physical reason of the observed variations of the solar granulation will come from numerical simulations. Therefore, the quiet sun does not appear to be immutable as claimed by Livingston \& Wallace (2003). This is an important piece of information even if quantitative results are still missing.

Acknowledgements. The authors acknowledge the support of the AustrianFrench scientific exchange programme "Amadeus" (R.M. and A.H.) and the support of the Austrian Fonds zur Förderung der Wissenschaftlichen Forschung (A.H. and M.S.). Part of this work was done while A.H. was visiting the Midi Pyrénées Observatory and R.M. the Institut für Geophysik, Astrophysik und Meteorologie, University Graz. R.M. acknowledges the technical staff of the Pic du Midi Observatory for their 20-year long support. All images were digitized with the Machine à Mesurer les Images Astronomiques (MAMA) at the
Observatoire de Paris. Special thanks are due to R. Chesnel, who made the measurements.

\section{References}

Basu, S., \& Antia, H. M. 2000, Solar Phys., 192, 449

Dziembowski, W. A., \& Goode, P. R. 2005, ApJ, 625, 548

Houdek, G., Chaplin, W. J., Appourchaux, J., et al. 2001, MNRAS, 327, 483

Kuhn, J. R. 2000, Space Sci. Rev., 94, 177

Li, L. H., Basu, S., Sofia, S., Robinson, F. J., \& Demarque, P. 2003, ApJ, 591, 1267

Livingston, W., \& Wallace 2003, Solar Phys., 212

Macris, C., \& Rösch, J. 1983, C. R. Acad. Sci. Paris, 296, 265

Macris, C., Muller, R., Rösch, J., \& Roudier, Th. 1984, in Small Scale Dynamical Processes in Quiet Stellar Atmospheres, ed. S. K. Keil, 265

Muller, R. 1988, Adv. Space Res., 8, 159

Roudier, Th. 1986, Thesis, Université Paul Sabatier, Toulouse, France

Sofia, S., Basu, S., Demarque, P., Li, L. H., \& Thuillier, G. 2005, ApJ, 632, L147 